\title{
Establishing a Research Agenda for Preventing Transmission of Multidrug-Resistant Organisms in Acute-Care Settings in the Veterans Health Administration
}

\author{
Eli N. Perencevich, MD, MS; ${ }^{1,2}$ Anthony D. Harris, MD, MPH; ${ }^{3,4}$ Christopher D. Pfeiffer, MD, MHS; ${ }^{5,6}$ Michael A. Rubin, \\ MD, PhD, MS; ${ }^{7,8}$ Jennifer N. Hill, MA; ${ }^{9}$ Gio J. Baracco, MD $;{ }^{10,11}$ Martin E. Evans, MD; ${ }^{12,13,14}$ J. Stacey Klutts, MD, PhD; ${ }^{1,15}$ \\ Judy A. Streit, MD; ${ }^{1,2}$ Richard E. Nelson, $\mathrm{PhD} ;{ }^{7,8}$ Karim Khader, PhD, MStat, MS; ${ }^{7,8}$ Heather Schacht Reisinger, $\mathrm{PhD}^{1,2}$
}

(See introductory commentary by Livorsi et al, pages 186-188.)

Transmission of multidrug-resistant organisms (MDRO) is a major contributor to the emergence of antibacterial resistant pathogens in healthcare settings. Transmission can occur through contact with contaminated environmental surfaces and other fomites or via the hands of healthcare workers. Numerous interventions have been implemented to target transmission prevention including hand hygiene surveillance and education, active surveillance, isolation of colonized or infected patients, patient cohorting, enhanced environmental cleaning, and the use of antimicrobial surfaces. Interventions can be generally divided into horizontal interventions that target the transmission of any organisms, such as hand hygiene, and vertical interventions that target a specific organism (eg, active surveillance and decolonization for methicillin-resistant Staphylococcus aureus [MRSA]). ${ }^{1}$

The Veterans Health Administration (VHA) has been a leader in transmission prevention within acute-care settings. A notable example is the 2007 MRSA directive that mandated that all acute-care units adopt a specific bundle of interventions to prevent the transmission of MRSA. ${ }^{2}$ The bundle consists of nasal surveillance testing for MRSA for all patients on admission, in-hospital transfer and discharge; contact precautions (CP) for MRSA colonized or infected patients; hand hygiene; and a change in the institutional culture that included institutional leadership with ground-level innovation along with hiring an MRSA coordinator at each facility. The implementation of this bundle has been associated with reduced MRSA acquisition and infection along with marked reduction in hospital-onset gram-negative bacteremia. ${ }^{3}$ In 2012, the VHA followed the example of the MRSA directive with a Clostridium difficile initiative that includes environmental management, hand hygiene, CP for the duration of diarrheal symptoms, and cultural transformation. ${ }^{4}$ Most recently, similar guidance for control of carbapenem-resistant Enterobacteriaceae (CRE) was released by the MDRO Prevention Office of the VHA National Infectious Diseases Service in September 2014, which was updated in January 2017. Given that the VHA is the largest integrated healthcare system in the United States and has a strong history of MDRO prevention and control, it is ideally positioned to further the generalizable knowledge around optimal control of MDRO transmission in both acute-care and long-term care settings.

In September 2016, a multidisciplinary group of investigators gathered to review the existing literature and outline a research agenda for transmission prevention in VHA. While the research targets were specifically designed to guide health services researchers in VHA, these suggested targets should be broadly useful in other settings including integrated healthcare systems outside the United States. A summary of the existing knowledge base, research recommendations and how needs fit within the VA Quality Enhancement Research Initiative (QUERI) 6-step model are provided in Table $1 .^{5}$

\section{RECOMMENDATIONS}

\section{Hand Hygiene}

Hand hygiene is the cornerstone of infection prevention efforts and the quintessential horizontal intervention. It is included in almost all infection control bundles from the

Affiliations: 1. Iowa City Veterans Affairs (VA) Health Care System, Iowa City, Iowa; 2. Department of Internal Medicine, University of Iowa Carver College of Medicine, Iowa City, Iowa; 3. Maryland VA Health Care System, Baltimore, Maryland; 4. Department of Epidemiology, School of Medicine, University of Maryland, Baltimore, Maryland; 5. Department of Hospital and Specialty Medicine, VA Portland Health Care System, Portland Oregon; 6. Department of Medicine, Oregon Health and Science University, Portland, Oregon; 7. VA Salt Lake City Health Care System, Salt Lake City, Utah; 8. Department of Medicine, University of Utah School of Medicine, Salt Lake City, Utah; 9. Department of Veterans Affairs, Center of Innovation for Complex Chronic Healthcare, Edward Hines Jr VA Hospital, Hines, Illinois; 10. Miami VA Health Care System, Miami, Florida; 11. Division of Infectious Diseases, Department of Medicine, University of Miami Miller School of Medicine, Miami Florida; 12. MRSA/MDRO Prevention Office, National Infectious Diseases Service, Patient Care Services, Veterans Health Administration, Washington, DC; 13. Lexington Veterans Affairs Medical Center, Lexington, Kentucky; 14. Division of Infectious Diseases, Department of Internal Medicine, University of Kentucky School of Medicine, Lexington, Kentucky; 15. Department of Pathology, University of Iowa Carver College of Medicine, Iowa City, Iowa.

Received November 7, 2017; accepted December 2, 2017

(c) 2018 by The Society for Healthcare Epidemiology of America. All rights reserved. 0899-823X/2018/3902-0009. DOI: 10.1017/ice.2017.309 
TA BLE 1. Proposed Veterans Healthcare Administration (VHA) Research Agenda for Transmission Prevention Research

\begin{tabular}{ll}
\hline Topic & Current Evidence \\
\hline Hand hygiene surveillance & $\begin{array}{c}\text { All hand hygiene opportunities are considered } \\
\text { equal risk. } \\
\text { The safe compliance threshold is unknown. }\end{array}$
\end{tabular}

The safe compliance threshold is unknown.

Inaccurate surveillance is generated by current direct-observation surveillance programs.

Limited data are available to support implementation of current automated surveillance systems.

Optimal methods and thresholds for hand hygiene compliance in outpatient settings are unknown.

Hand hygiene improvement interventions

Active surveillance
Current hand hygiene improvement bundles include education, reminders, feedback, administrative support and access to alcohol-based hand rubs, but the sustainability of interventions is limited.

Existing hand hygiene preparation are short acting and must be reapplied before and after each opportunity.

Active surveillance is typically bundled with other infection control practices including CP-based isolation or decolonization. The role of active surveillance in ICU settings has received the most attention, but data examining the role in short-stay acute-care hospitals including critical access hospitals, long-term acute-care facilities, and nursing homes (community living centers) are limited.

"Targeted" active surveillance, or performing surveillance on subgroups suspected of a higher risk of colonization, has little supportive evidence in the literature. Several studies have looked at prediction of colonization for individual MDROs, but few studies of targeted implementation have evaluated the benefits and cost-effectiveness.

The utility of active surveillance for certain emerging pathogens has been limited by the ability to culture or detect the organism (eg, Clostridium difficile) and the rarity of the organism in VHA settings (eg, CRE).

Active surveillance is pathogen specific and will identify many patients colonized with pathogens they are not likely to transmit. Other patients may be "super-spreaders" because they have conditions or are colonized with pathogens that make them more likely to transmit the organism.

Active surveillance for $S$. aureus and MRSA when combined with decolonization and modifications to perioperative antibiotic regimens appears to be highly effective and safe, particularly in orthopedic and cardiothoracic surgical settings, yet adoption of this approach in VHA is limited.
Research Needs (QUERI Step Model)

Determine which hand hygiene opportunities are associated with highest transmission risk (step 2)

Conduct prospective cohort studies to examine the association between hand hygiene compliance and HAI rates, including multidrug-resistant organism (MDRO) acquisition (steps 2 and 3)

Quantify the size of the Hawthorne effect (step 3) and develop and validate novel direct-observation surveillance methods that reduce reporting bias and the Hawthorne effect (step 4)

Validate the effectiveness and cost-effectiveness of automated surveillance systems (steps 5 and 6)

Conduct basic epidemiological investigations exploring transmission risks outside of acute-care settings and hand hygiene's role in interrupting transmission (steps 2 and 3)

Develop, test, and validate sustainable interventions through cluster-randomized controlled trials, well-designed quasi-experimental studies; consider behavioral (eg, peer comparison) or behavioral economic interventions (steps 4, 5, and 6)

Conduct pilot tests of effectiveness of long-acting preps including studies to evaluate the barriers to implementing long-acting agents (step 4)

Establish prevalence thresholds for cost-effectiveness of active surveillance across various pathogens and clinical settings when combined with $\mathrm{CP}$, decolonization or both; include cohort studies, quasi-experimental studies, and decision-analytic models (steps 2 and 3)

Validate the effectiveness and cost-effectiveness of prediction rule guided active surveillance using prospective cohort studies; study specific organisms including MRSA, CRE, and CDI; given the expense of the current VHA MRSA bundle, conduct a trial comparing a targeted approach to the existing bundle could be valuable (steps 4,5 , and 6 )

Examine approaches for including active surveillance in the existing CDI bundle across care settings (eg, ICU); perform multicenter cohort or quasi-experimental studies to determine VHA-specific parameter estimates and inform power calculations prior to modeling or large-scale cluster-randomized trials (steps 4, 5, and 6)

Develop and validate prediction rules and risk stratification methods to improve the effectiveness and cost-effectiveness of active surveillance methods (step 4)

Perform hybrid effectiveness-implementation studies in cardiac, orthopedic, and trauma surgical settings; explore the effectiveness of this approach in other surgical procedures and other clinical settings (eg, hemodialysis) (steps 4, 5, and 6) 


\begin{tabular}{|c|c|}
\hline Topic & Current Evidence \\
\hline \multirow[t]{3}{*}{$\begin{array}{l}\text { Isolation measures: } \mathrm{CP} \text {, } \\
\text { universal gloving, and } \\
\text { patient cohorting }\end{array}$} & $\begin{array}{l}\text { Existing literature supporting the efficacy, cost- } \\
\text { effectiveness, and risk-benefit ratio of } \mathrm{CP} \text {, univers } \\
\text { gowning/gloving, universal gloving, and patient } \\
\text { cohorting are limited and inconclusive. }\end{array}$ \\
\hline & $\begin{array}{l}\text { The importance of infections that manifest } \\
\text { postdischarge from acute-care hospitals has been } \\
\text { underrecognized in existing studies. }\end{array}$ \\
\hline & $\begin{array}{l}\text { Epidemiological evidence suggests that healthcare } \\
\text { workers become contaminated when caring for } \\
\text { MDRO-positive patients, but the role of limiting } \\
\text { contamination in reducing patient-to-patient } \\
\text { transmission is less well defined. }\end{array}$ \\
\hline
\end{tabular}

The statistical validity of existing studies assessing the benefits of isolation strategies and discontinuation of $\mathrm{CP}$ is poor.

Contact precautions and patient isolation have been associated with noninfectious physical and psychological adverse events, yet studies have been limited by selection bias and confounding. Newer well-controlled and randomized studies have failed to find an association. Existing literature does not support completion of a meta-analysis.

Similar to active surveillance, data establishing thresholds for facility-wide, unit-wide, or individual-level targeted use of isolation measures are limited.

Adherence to $\mathrm{CP}$, similar to hand hygiene, remains poor.

Benchmarks for cleanliness after daily or terminal cleaning have not been definitively established or are linked to reduced transmission rates.

The role of daily room cleaning is known to be important but is underappreciated.
Research Needs (QUERI Step Model)

Large clinical trials measuring the effectiveness and costeffectiveness of isolation measures are needed but are limited by the need for large sample sizes, collection of admission and discharge surveillance cultures, and tracking of postdischarge infections (step 2).

Conduct cohort studies utilizing VHA databases to estimate the incidence and impact of postdischarge infections for those who acquire MRSA, CDI, or MDR-gram negative bacilli and to demonstrate the value of CP (step 1)

Estimate the transmission that occurs from contaminated healthcare workers to uncolonized patients by completing prospective cohort studies with surveillance cultures and whole-genome or other sequencing methods; alternatively, simulate transmission using noninfectious DNA markers (eg, cauliflower mosaic virus) to estimate MDRO spread (step 2)

Develop statistical guidelines outlining sample size needs for all transmission-based interventions including $\mathrm{CP}$, universal gloving, cohorting, and hand hygiene (step 4)

Complete additional high-quality observational or randomized studies or trials embedded in other trials that examine the association between CP or patient isolation and physical or psychological adverse events (steps 5 and 6)

Utilize existing MRSA and CDI admission prevalence, importation and acquisition rates, and HAI/ postdischarge infection rates to populate existing mathematical models to guide VHA and individual facilities in determining high-risk populations where implementing active surveillance and isolation will be most cost-effective (steps 4,5 , and 6 )

In parallel with studies described above for hand hygiene, conduct similar studies aimed at improving the compliance and reducing the burden of $\mathrm{CP}$ are needed, eg, examine the noninferiority of "safe zones" in ICU and non-ICU settings where healthcare workers can enter nonisolated patient rooms without donning gowns or gloves (steps 4, 5, and 6)

Conduct epidemiological investigations to evaluate the association between levels of environmental contamination and patient acquisition (ie, MRSA or CDI) by leveraging existing VA surveillance and administrative data; use the parameters collected to populate mathematical models structured to estimate safe environmental cleanliness thresholds (steps 2 and 3)

Determine which in-room surfaces are the highestvalue targets for decontamination (step 2)

Use mathematical models to gain insight regarding the effectiveness and cost-effectiveness of enhanced daily cleaning strategies in ICUs and other care settings (steps 2 and 3) 


\begin{tabular}{|c|c|c|}
\hline Topic & Current Evidence & Research Needs (QUERI Step Model) \\
\hline & $\begin{array}{l}\text { Recognition of role that the prior room occupant } \\
\text { plays in acquisition of MDRO has led to widespread } \\
\text { adoption of enhanced terminal cleaning } \\
\text { technology, including ultraviolet-C and hydrogen } \\
\text { peroxide devices, despite limited proven clinical } \\
\text { benefit. }\end{array}$ & $\begin{array}{l}\text { Considering widespread adoption of enhanced } \\
\text { terminal cleaning devices in VHA, conduct a quasi- } \\
\text { experimental study comparing healthcare-associated } \\
\text { infection (HAI) and MDRO infection rates before/ } \\
\text { after adoption of the technology with concurrent } \\
\text { nonequivalent controls (steps } 5 \text { and 6) }\end{array}$ \\
\hline & $\begin{array}{l}\text { Barriers to enhanced daily and terminal cleaning exist } \\
\text { including optimal methods for audit and feedback } \\
\text { such as specific methods for monitoring } \\
\text { compliance. }\end{array}$ & $\begin{array}{l}\text { In studies examining the sensitivity and specificity of } \\
\text { monitoring methods, incorporate qualitative } \\
\text { investigations examining barriers and facilitators } \\
\text { of implementation across the care continuum } \\
\text { (steps } 5 \text { and } 6 \text { ) }\end{array}$ \\
\hline
\end{tabular}

NOTE. QUERI, Quality Enhancement Research Initiative; HAI, hospital-associated infection; MDRO, multidrug-resistant organism; CP, contact precautions; MRSA, methicillin-resistant Staphylococcus aureus; CRE, carbapenem-resistant Enterobacteriaceae; CDI, Clostridium difficile infection, VHA, Veterans Health Administration; ICU, intensive care unit.

central-line-associated bloodstream infection (CLABSI) bundle to the VHA MRSA bundle., ${ }^{2,6}$ Yet despite all the attention, hand hygiene compliance remains dismal, with the best estimates of compliance ranging from $34 \%$ to $57 \% .{ }^{7}$ Hand hygiene initiatives can be broken down into 2 major components: surveillance and interventions. Specific interventions are listed in Table 1.

Surveillance. Surveillance of hand hygiene practice is the cornerstone of hand hygiene programs, and audit and feedback of accurate compliance rates are the most basic components of hand hygiene improvement. However, the difficulty in hand hygiene surveillance can be summarized in this quote from Dr Mark Chassin, President and Chief Executive Officer of The Joint Commission: "It's interesting that a number of the hospitals were misled by faulty data to believe that they were doing as well as, say, $85 \%$, at baseline rather than (a more accurate) 48\%." Given that the Joint Commission could not attain compliance rates above $82 \%$ under study conditions and no longer requires a specific hand hygiene target be met in their National Patient Safety Goals, an evidence-based hand hygiene compliance target or "safe threshold" is needed in acute-care, critical-care, and long-term care settings. Thus, the processes that go into hand hygiene surveillance need critical analysis and innovative studies. First, the current gold standard for measuring hand hygiene compliance is direct observation, which has inherent biases such as the Hawthorne effect. ${ }^{9}$ Research related to best practices for direct observation, including multicenter VA studies, is appearing; however, much more research is needed to insure that infection prevention and control teams have accurate hand hygiene surveillance data. ${ }^{10}$ Some call for automated systems, but more technological developments and research are needed to fully realize the potential of this type of surveillance, including randomized control or quasi-experimental studies to validate the systems and costeffectiveness studies. ${ }^{11}$ Critical analysis is also needed regarding defining hand hygiene opportunities-the denominator of hand hygiene compliance rates. For example, hand hygiene might not be necessary prior to donning gloves. ${ }^{12}$ Thus, eliminating this opportunity or others might reduce the denominator for calculating compliance rates and focus the attention of the clinical staff and administrative oversight on the most critical hand hygiene opportunities. Additional attention is needed in developing optimal surveillance methods for non-acute-care settings, including outpatient clinics.

Hand hygiene improvement interventions. Beyond audit and feedback, the existing World Health Organization, Centers for Disease Control and Prevention (CDC), and VHA hand hygiene bundles have been shown to increase hand hygiene compliance in acute-care settings. ${ }^{13}$ These bundles have included education, reminders, administrative support, and access to alcohol-based hand rub and have typically been evaluated using single-center quasi-experimental studies. Novel and sustainable interventions are still needed and might include long-acting hand hygiene preparation. Any evaluation of long-acting agents would need to include qualitative evaluation to understand how implementation might impact overall behavior. Evaluation of existing bundle components and novel interventions should occur using cluster-randomized trials or well-designed multicenter quasiexperimental studies using factorial designs to determine the least number of components required to maintain compliance over established safe thresholds without overburdening clinical staff. Intervention studies could include longer postintervention observational periods to determine which 
interventions most effectively sustain compliance rates above safe thresholds. Interventions that sustain compliance may differ from those that initially increase compliance.

\section{Active Surveillance}

Active surveillance, obtaining screening tests of patients not known to be colonized or infected with a specific organism, has played an integral role in the control of MDRO for decades. Active surveillance and subsequent isolation and/or decolonization of patients carrying a single pathogen of interest is the prototypical vertical intervention. ${ }^{1}$ Passive surveillance, which typically relies on clinical cultures, misses or delays the detection of the majority of patients who could potentially spread the pathogen to uncolonized patients. ${ }^{14}$ Yet the role of active surveillance has not been without controversy, particularly after the 2006 CDC Healthcare Infection Control and Prevention Advisory Committee guidelines suggested that facilities could choose to apply active surveillance in a targeted fashion. ${ }^{15}$ Early evidence supported a role for active surveillance in national control efforts for MRSA in the Netherlands and other countries ${ }^{16}$, in regional vancomycinresistant enterococci control, ${ }^{17}$ and in numerous outbreak settings. However, more recent clinical trials have been less supportive when active surveillance was combined with delayed implementation of CP for VRE and MRSA ${ }^{18}$ or when compared to universal decolonization. ${ }^{19}$

In the VHA, active surveillance has played a central and successful role in the MRSA bundle adopted in $2007^{2}$ but was not included in the subsequent $C$. difficile infection (CDI) bundle. The VHA CRE bundle recommended targeted screening of high-risk patients or patients sharing a room with CREpositive patients based on individual facility's risk assessment. Specific recommendations are provided in Table 1. Potential targets for future study include (1) establishing organism-specific (eg, CRE) and setting-specific (eg, intensive care unit [ICU]) thresholds for cost-effectiveness of active surveillance, (2) developing and validating prediction-rule guided active surveillance, (3) exploring and studying the inclusion of active surveillance or targeted active surveillance into existing CDI and CRE VHA directives, and (4) completing implementationeffectiveness studies of MRSA/S. aureus screening plus decolonization bundles in surgical and nonsurgical settings.

\section{Isolation Measures}

Isolation measures include $\mathrm{CP}$ and patient cohorting. Contact precautions require healthcare workers to wear gowns and gloves when entering patient rooms and caring for patients. Cohorting requires that patients colonized or infected with an MDRO be placed in the same unit, together in a shared room, or in single-occupancy rooms. Contact precautions can be implemented universally for all patients, ${ }^{20}$ selectively as guided by active surveillance interventions as used in the VHA MRSA directive, or passively for patients known to be currently or historically infected with select MDROs. Some facilities also use syndrome-based CP (ie, isolation of patients with draining wounds or uncontrolled diarrhea). The additional benefit of gowns when added to gloves has not been well established, and recent studies have supported potential benefits of universal gloving strategies. ${ }^{21}$

Recently, the utility of CP, particularly when combined with active surveillance programs, has been questioned. ${ }^{22}$ Despite this, $\mathrm{CDC}$ guidance has remained unchanged on this issue, and most infection control programs in the United States continue to utilize CP. ${ }^{15,23}$ Continued utilization of $\mathrm{CP}$ will require additional large-scale studies to establish the benefits, potential harms, and costs associated with targeted or universal implementation for endemic (eg, MRSA) and emerging (eg, CRE) pathogens. Existing studies have been predominantly performed in ICU settings and the utility of CP in non-ICU and long-term care settings is understudied. Additionally, the utility of CP independent of other, often bundled interventions, such as cohorting and enhanced environmental cleaning, needs to be investigated. ${ }^{24}$

\section{Enhanced Environmental Cleaning Interventions}

Until recently, the focus of infection control has been on the individual patient and not the environment surrounding the patient. However, it is well recognized that pathogens can be transmitted from porous (eg, textiles) or hard surfaces (eg, tables) directly or indirectly via contaminated hands of healthcare workers or patients. ${ }^{25}$ Thus, daily environmental cleaning and disinfection and terminal cleaning (ie, at patient discharge) are important components in transmission prevention.

Few studies have examined the risks and benefits of daily environmental cleaning while the MDRO-colonized or infected patient remains in the room. Given that most healthcare worker contacts with the environment occur during this period, additional studies are needed to outline the risks of environmental contamination during this period and the methods for monitoring and reducing the risk through daily or more frequent cleaning or disinfection. Novel technologies, such as antimicrobial textiles (eg, privacy curtains or surgical scrubs) and antimicrobial surfaces (eg, copper coatings), have been evaluated as methods for continuous cleaning with mixed success. ${ }^{26-30}$

The current focus on environmental cleaning and disinfection has been on terminal cleaning at patient discharge based on the recognition that patients who inhabit a room whose prior room occupant carried an MDRO might be at higher risk for acquiring the same pathogen. ${ }^{31}$ Terminal cleaning methods include surface cleaning using chemical disinfectants (eg, quaternary ammonium or bleach) and no-touch automated systems (eg, disinfecting ultraviolet [UV-C] light and hydrogen peroxide vapor). The effectiveness and cost-effectiveness of these technologies are currently being evaluated and would benefit from more in-depth comparative effectiveness studies. ${ }^{32}$ 
Technologies for monitoring environmental cleaning and disinfection have been evaluated including fluorescent UV surface markers and adenosine triphosphate bioluminescence. However, frequency of monitoring, intensity of monitoring (ie, number of surfaces tested), and the implementation of monitoring bundles have not been adequately evaluated. A recent review highlighted the importance of contextual factors, and further studies, particularly outside acute-care facilities, are needed. ${ }^{33}$ Finally, while environmental cleaning technologies have been linked to reduced environmental bioburden, few studies have been able to link interventions to improved clinical outcomes such as reductions in MDRO infections. The reasons include rarity of outcomes and many potential confounders in the pathway between a contaminated environment and clinical infection, including unit-level hand hygiene compliance and patient comorbidity. Thus, without large and cost-prohibitive cluster-randomized trials, a definitive link between environmental cleaning and improved clinical outcomes may never be established. Mathematical models that incorporate the best estimates of intervention benefits and other important parameters, such as hand hygiene compliance, are likely our best hope in estimating the effectiveness and cost-effectiveness of old and new technologies. ${ }^{34,35}$

\section{ACKNOW LEDGMENTS}

This work was supported in part by funding from the VA Health Services Research and Development (HSR\&D) Service Center of Innovation (COIN) conference supplement for ("Setting the Clinical Research Agenda for MDROs in VA," grant no. CIN 13-412), and VA Quality Enhancement Research Initiative CARRIAGE Program (grant no. IP1 HX001993-01A1). The views expressed in this article are those of the authors and do not necessarily reflect the position or policy of the Department of Veterans Affairs or the US government. All authors report no conflicts of interest or financial disclosures relevant to this article.

Address correspondence to Eli N. Perencevich, MD, Center for Comprehensive Access and Delivery Research and Evaluation (CADRE), Iowa City VA Health Care System, Department of Internal Medicine, University of Iowa Carver College of Medicine, 601 Highway 6 West Iowa City, IA 52246 (eli-perencevich@uiowa.edu).

\section{REFERENCES}

1. Wenzel RP, Edmond MB. Infection control: the case for horizontal rather than vertical interventional programs. Int $J$ Infect Dis 2010;14:S3-S5.

2. Jain R, Kralovic SM, Evans ME, et al. Veterans Affairs initiative to prevent methicillin-resistant Staphylococcus aureus infections. N Engl J Med 2011;364:1419-1430.

3. Goto M, O'Shea AMJ, Livorsi DJ, et al. The effect of a nationwide infection control program expansion on hospital-onset gramnegative rod bacteremia in 130 Veterans Health Administration medical centers: an interrupted time-series analysis. Clin Infect Dis 2016;63:642-650.

4. Evans ME, Kralovic SM, Simbartl LA, Jain R, Roselle GA. Effect of a Clostridium difficile infection prevention initiative in Veterans Affairs acute care facilities. Infect Control Hosp Epidemiol 2016;37:720-722.
5. Stetler CB, Mittman BS, Francis J. Overview of the VA Quality Enhancement Research Initiative (QUERI) and QUERI theme articles: QUERI series. Implement Sci 2008;3:8.

6. Marschall J, Mermel LA, Fakih M, et al. Strategies to prevent central line-associated bloodstream infections in acute care hospitals: 2014 update. Infect Control Hosp Epidemiol 2014; 35:753-771.

7. Kingston L, O'Connell NH, Dunne CP. Hand hygiene-related clinical trials reported since 2010: a systematic review. J Hosp Infect 2016;92:309-320.

8. Berman S. An interview with Mark Chassin. Jt Comm J Qual Patient Saf 2010;36:465-469.

9. Chen LF, Vander Weg MW, Hofmann DA, Reisinger HS. The Hawthorne effect in infection prevention and epidemiology. Infect Control Hosp Epidemiol 2015;36:1444-1450.

10. Yin J, Reisinger HS, Vander Weg M, et al. Establishing evidence-based criteria for directly observed hand hygiene compliance monitoring programs: a prospective, multicenter cohort study. Infect Control Hosp Epidemiol 2014;35: 1163-1168.

11. Ward MA, Schweizer ML, Polgreen PM, Gupta K, Reisinger HS, Perencevich EN. Automated and electronically assisted hand hygiene monitoring systems: a systematic review. Am J Infect Control 2014;42:472-478.

12. Rock C, Harris AD, Reich NG, Johnson JK, Thom KA. Is hand hygiene before putting on nonsterile gloves in the intensive care unit a waste of health care worker time? A randomized controlled trial. Am J Infect Control 2013;41: 994-996.

13. Schweizer ML, Reisinger HS, Ohl M, et al. Searching for an optimal hand hygiene bundle: a meta-analysis. Clin Infect Dis 2014;58:248-259.

14. Perencevich EN, Fisman DN, Lipsitch M, Harris AD, Morris JG Jr, Smith DL. Projected benefits of active surveillance for vancomycin-resistant enterococci in intensive care units. Clin Infect Dis 2004;38:1108-1115.

15. Siegel JD, Rhinehart E, Jackson M, Chiarello L, Healthcare Infection Control Practices Advisory C. Management of multidrug-resistant organisms in health care settings, 2006. Am J Infect Control 2007;35:S165-S193.

16. Bootsma MC, Diekmann O, Bonten MJ. Controlling methicillinresistant Staphylococcus aureus: quantifying the effects of interventions and rapid diagnostic testing. Proc Natl Acad Sci USA 2006;103:5620-5625.

17. Ostrowsky BE, Trick WE, Sohn AH, et al. Control of vancomycin-resistant enterococcus in health care facilities in a region. N Engl J Med 2001;344:1427-1433.

18. Huskins WC, Huckabee CM, O'Grady NP, et al. Intervention to reduce transmission of resistant bacteria in intensive care. $N$ Engl J Med 2011;364:1407-1418.

19. Huang SS, Septimus E, Kleinman K, et al. Targeted versus universal decolonization to prevent ICU infection. $N$ Engl J Med 2013;368:2255-2265.

20. Harris AD, Pineles L, Belton B, et al. Universal glove and gown use and acquisition of antibiotic-resistant bacteria in the ICU: a randomized trial. JAMA 2013;310:1571-1580.

21. Yin J, Schweizer ML, Herwaldt LA, Pottinger JM, Perencevich EN. Benefits of universal gloving on hospital-acquired infections in acute care pediatric units. Pediatrics 2013;131: e1515-e1520. 
22. Morgan DJ, Wenzel RP, Bearman G. Contact precautions for endemic MRSA and VRE: time to retire legal mandates. JAMA 2017;318:329-330.

23. Morgan DJ, Murthy R, Munoz-Price LS, et al. Reconsidering contact precautions for endemic methicillin-resistant Staphylococcus aureus and vancomycin-resistant Enterococcus. Infect Control Hosp Epidemiol 2015;36:1163-1172.

24. Fitzpatrick F, Perencevich EN. Putting contact precautions in their place. J Hosp Infect 2017;96:99-100.

25. Samore MH, Venkataraman L, DeGirolami PC, Arbeit RD, Karchmer AW. Clinical and molecular epidemiology of sporadic and clustered cases of nosocomial Clostridium difficile diarrhea. Am J Med 1996;100:32-40.

26. Anderson DJ, Addison R, Lokhnygina Y, et al. The Antimicrobial Scrub Contamination and Transmission (ASCOT) trial: a threearm, blinded, randomized controlled trial with crossover design to determine the efficacy of antimicrobial-impregnated scrubs in preventing healthcare provider contamination. Infect Control Hosp Epidemiol 2017;38:1147-1154.

27. Burden M, Keniston A, Frank MG, et al. Bacterial contamination of healthcare workers' uniforms: a randomized controlled trial of antimicrobial scrubs. J Hosp Med 2013;8:380-385.

28. Harbarth S, Maiwald M, Dancer SJ. The environment and healthcare-acquired infections: why accurate reporting and evaluation of biological plausibility are important. Infect Control Hosp Epidemiol 2013;34:996-997.

29. Salgado CD, Sepkowitz KA, John JF, et al. Copper surfaces reduce the rate of healthcare-acquired infections in the intensive care unit. Infect Control Hosp Epidemiol 2013;34:479-486.
30. Schweizer M, Graham M, Ohl M, Heilmann K, Boyken L, Diekema D. Novel hospital curtains with antimicrobial properties: a randomized, controlled trial. Infect Control Hosp Epidemiol 2012;33:1081-1085.

31. Martinez JA, Ruthazer R, Hansjosten K, Barefoot L, Snydman DR. Role of environmental contamination as a risk factor for acquisition of vancomycin-resistant enterococci in patients treated in a medical intensive care unit. Arch Intern Med 2003;163:1905-1912.

32. Anderson DJ, Chen LF, Weber DJ, et al. Enhanced terminal room disinfection and acquisition and infection caused by multidrug-resistant organisms and Clostridium difficile (the Benefits of Enhanced Terminal Room Disinfection study): a cluster-randomised, multicentre, crossover study. Lancet 2017;389:805-814.

33. Han JH, Sullivan N, Leas BF, Pegues DA, Kaczmarek JL, Umscheid CA. Cleaning hospital room surfaces to prevent health care-associated infections: a technical brief. Ann Intern Med 2015;163:598-607.

34. Barnes SL, Morgan DJ, Harris AD, Carling PC, Thom KA. Preventing the transmission of multidrug-resistant organisms: modeling the relative importance of hand hygiene and environmental cleaning interventions. Infect Control Hosp Epidemiol 2014;35:1156-1162.

35. Nelson RE, Jones $M$, Leecaster $M$, et al. An economic analysis of strategies to control Clostridium difficile transmission and infection using an agent-based simulation model. PLoS One 2016; 11:e0152248. 